\title{
Impact of Single Dose Praziquantel Treatment on Schistosoma haematobium Infection among School Children in an Endemic Nigerian Community
}

\author{
Babatunde Adewale,*, Margaret A. Mafe', Medinat A. Sulyman', Emmanuel T. Idowu², Morakinyo B. Ajayi', \\ David O. Akande', James H. Mckerrow ${ }^{3}$, Emmanuel O. Balogun ${ }^{4}$ \\ ${ }^{1}$ Public Health and Epidemiology Department, Nigerian Institute of Medical Research P.M.B 2013 Yaba, Lagos, Nigeria; ${ }^{2}$ Zoology Department, \\ Faculty of Science, University of Lagos, P.M.B 1031 Akoka, Lagos, Nigeria; ${ }^{3}$ Center for Discovery and Innovation in Parasitic Diseases, Skaggs \\ School of Pharmacy and Pharmaceutical Sciences, University of California San Diego, La Jolla, California, United States of America; ${ }^{4}$ Department of \\ Biochemistry, Ahmadu Bello University, Zaria 2222, Kaduna State, Nigeria
}

\begin{abstract}
Schistosomiasis is prevalent in Nigeria, and the foremost pathogen is Schistosoma haematobium, which affects about 29 million people. Single dose of the drug praziquantel is often recommended for treatment but the efficacy has not been documented in certain regions. Therefore, this study was designed to assess the impact of single dose praziquantel treatment on S. haematobium infection among school children in an endemic community of South-Western Nigeria. Urine samples were collected from 434 school children and $10 \mathrm{ml}$ was filtered through Nucleopore filter paper before examination for egg outputs by microscopy. The prevalence was $24.9 \%$ at pre-treatment. There was no statistically significant difference for the prevalence of infection between males (14.7\%) and females (10.2\%), although the mean egg count for the females $(9.87)$ was significantly more $(P<0.05)$ than the males $(6.06)$. At 6 and 12 months post-treatment there was $74.4 \%$ and $86.4 \%$ reduction in the mean egg count, respectively. Interestingly, an increased prevalence of infection from $2.1 \%$ at 6 months to $7.7 \%$ at 12 months post-treatment was observed, nonetheless the mean egg count was reduced to 0.27 at 12th month from 1.98 at 6 months post-treatment. Resurgence in the prevalence rate between 6 and 12 months post-treatment with praziquantel is herein reported and the need for a follow-up treatment in endemic areas for adequate impact on schistosomiasis control is discussed.
\end{abstract}

Key words: Schistosoma haematobium, school, children, praziquantel

\section{INTRODUCTION}

The presence of Schistosoma haematobium and Schistosoma mansoni in Nigeria has been documented since 1881 from accounts of the German traveler, Nachtigal who passed through the eastern region of Borno [1]. According to the report, it was introduced by Fulani herdsmen arriving from the Upper Nile valley [1]. Today, Schistosomiasis is recognized as one of the most devastating neglected tropical diseases (NTDs) in sub-Saharan Africa, and it is estimated that 779 million people are atrisk of infection while about 250 million people are currently infected $[2,3]$ producing a disease burden that could exceed that

- Received 25 May 2018, revised 6 November 2018, accepted 12 November 2018.

*Corresponding author (badewale@hotmail.com)

(c) 2018, Korean Society for Parasitology and Tropical Medicine

This is an Open Access article distributed under the terms of the Creative Commons

Attribution Non-Commercial License (http://creativecommons.org/licenses/by-nc/4.0) which permits unrestricted non-commercial use, distribution, and reproduction in any

medium, provided the original work is properly cited. of malaria, which according to the Global Disease Burden study of 2010 was 3.31 million disability-adjusted life years (DALYs) and 11,700 deaths per year [3-6]. More worrisome is the fact that female genital schistosomiasis is a co-factor in the transmission of HIV [7,8], and Nigeria is categorized as a HIV endemic country. Therefore, schistosomiasis continues to be of great public health significance in Nigeria, and the epidemiology varies greatly within and between regions of the country [9]. Nigeria has the greatest number of cases of schistosomiasis worldwide, with both intestinal schistosomiasis caused by S. mansoni and the urogenital schistosomiasis caused by $S$. haematobium endemic in the country [10]. Currently, praziquantel is the major focus of preventive chemotherapy which is delivered by mass drug administration to those shown to be, or presumed, to be at-risk of infection and the disease [4]. Provision of praziquantel which cost just a few cents per tablet when given yearly to children could avert a level of morbidity in Africa that exceeds that of malaria or other disease scourges [11]. The realiza- 
tion of this goal can only be attainable with the identification of all endemic communities such as Ipogun community in Nigeria for mass treatment of schistosomiasis and other soil transmitted helminthic infections, especially that these diseases affect mainly the agrarian and rural populations [12]. As of 2006, a majority of Nigerians lack access to safe water coverage (53\%) and sanitation (70\%), a situation that has largely remainunchanged [13-15]. Praziquantel is the mainstay of the current strategy recommended by WHO against schistosomiasis for morbidity control through preventive chemotherapy $[4,16,17]$. Successful control programmes in China, Egypt, Brazil, Philipines, Japan and Uganda show that treating the total eligible population in areas that are highly endemic for schistosomiasis with praziquantel for a few years results in substantial reductions in the indices (e.g., prevalence, parasite intensity, and anaemia) of the disease and in the amount of maintenance drug required [18]. Despite WHO member states endorsement of the WHA 65.21 resolution on elimination of schistosomiasis [4] there is the need to identify pockets of infections as well as the effectiveness of praziquantel through the adoption of the right treatment strategy which may vary from one country to another depending on the community level of infrastructural development, health promotion and education. Yet over a decade after a World Health Assembly's resolution to provide complete drug coverage for schistosomiasis, less than $10 \%$ of school-aged children at risk received praziquantel $[17,19]$. This present study which was a longitudinal study evaluates the effectiveness of praziquantel in the treatment of schistosomiasis and also to monitor possible development of resistance in the use of praziquantel for the control of schistosomiasis.

\section{MATERIALS AND METHODS}

\section{Study area}

The study was conducted in Ipogun community in Ondo state of Nigeria. It is situated in the tropical rainforest belt of Western Nigeria. It is a rural community that enjoys electricity and it lies between latitude $5^{\circ} 6^{\prime} \mathrm{N}$ and $8^{\circ} 2^{\prime} \mathrm{N}$ and longitude $4^{\circ} 17^{\prime} \mathrm{E}$ and $6^{\circ} 17^{\prime} \mathrm{E}$. There are 4 elementary schools and 1 secondary school in the community. Three of the four elementary schools are government owned schools while one is a private school. The inhabitants of the community are mainly farmers. There are very few water wells in the community thereforeresidents depend mainly on river Apogun which traverses the community for their water supply.

\section{Consent and ethical approval}

Prior to commencement of the study administrative approval was obtained from the State Ministry of Health through a letter and the Local Government Primary Health Care Department during advocacy visit. Ethical approval for the protocol was obtained from the Institutional Review Board of Nigerian Institute of Medical Research (IRB/14/237). The community head was duly informed and the parents were adequately enlightened on the purpose of the study to obtain their verbal consent and assent of the children. All the head teachers and teachers of the schools were adequately enlightened and mobilized for the study prior to commencement.

\section{Study design}

The inclusion criteria were: children who enrolled in the study school; child must assent to participate. The exclusion criteria were: children who did not enroll in the study school; child who did not assent to participate.

All the participants (age range 5-18 years old) were enrolled in the survey according to the school enrollment list in the class register. Each participant was given a survey code which included the school code, class and serial number which was the same as the code on the specimen containers for urine samples. Other demographic data such as sex and age of each child was recorded during the enrollment for the survey. Urine samples were collected from all the participants that consented to participate in the study between 10.00 and $14.00 \mathrm{hr}$ [20]. The frequency of visible haematuria was also recorded. Filtration technique using nucleopore filter papers with swinnex filter holder for $10 \mathrm{ml}$ of urine and microscopy was employed in examining the nucleopore filter papers for the presence and the intensity of $S$. haematobium eggs which was recorded as egg output per $10 \mathrm{ml}$ of urine [20]. All the recruited participants were treated with single oral dose of praziquantel $(600 \mathrm{mg}$, Batch No: M70116) at a dose of $40 \mathrm{mg} / \mathrm{kg}$ body weight immediately after submitting urine samples by directly observed treatment. Urine samples of the participants which were processed as described above were examined at pre-treatment, 6 and 12 months post-treatment intervals to determine changes in parasites intensities and prevalence with time.

\section{Data analysis}

Demographic data and results from the examined samples were analyzed using IBM SPSS version 20 (SPSS, Chicago, Illinois, USA). Results were presented using descriptive statistics 
in frequencies, percentages and charts. Inferential statistics was also performed using chi square to test the significance of variation of infection between age group, sex and schools were tested by chi-square tests.

\section{RESULTS}

A total of 434 children (male 56.2\%; female 43.8\%) were examined for $S$. haematobium egg (Fig. 1) infection at baseline in the 4 elementary schools in the community with ages ranging from 5 to 18 years and mean age $9.16 \pm 2.45$. At the end of 12 months we had $95.6 \%$ of the children still in the study. The drop-out rate of the children for the period of 12 months was $4.4 \%$ being the total number of children that were not present till the end of the survey at 12 months.

The prevalence of infection at pre-treatment was $24.9 \%$ (Males 14.7\%; Females 10.2\%) although there was no statistically significant difference $(P>0.05)$ in infection rate between male and female. The mean urine egg count of the samples col-

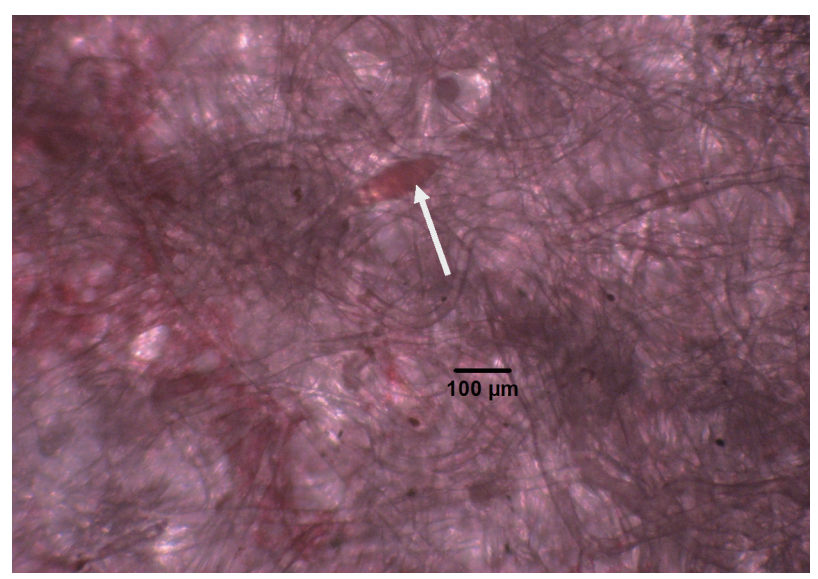

Fig. 1. Egg of Schistosoma haematobium (arrow), stained with eosin on Whatman fiter paper. An estimated size is $175 \times 60 \mu \mathrm{m}$. lected from female students (9.87) was greater than that of the males (6.06). Age group 9-10 years had the highest prevalence of $30.5 \%$, followed by age group 7-8 years with a prevalence of $29.5 \%$ while age group 13-14 years had the lowest prevalence of infection (Table 1). There was significant difference in the prevalence of infection among the age groups $(P<0.05)$.

Prevalence of infection by school ranged from $14 \%$ to $46.9 \%(P<0.001)$. Prevalence of infection among the participants at 6 months post treatment was $2.1 \%$ ( 9 children) which showed a $91.6 \%$ reduction in prevalence. Among the 9 infected participants, 6 were new infections while the remaining 3 were part of those infected at pre-treatment. Age groups $7-8$ and $9-10$ had the highest prevalence of $0.7 \%$ each. Females had a higher prevalence of infection $6(1.4 \%)$ at 6 months post treatment when compared to males $3(0.7 \%)$ though the difference was not statistically significant $(P>0.05)$. The prevalence of infection among the participants at 12 months post treatment (7.7\%) showed an increase in prevalence over the 6 months prevalence $(2.1 \%)$ with a total of $32(7.7 \%)$ participants infected at 12 months which was $69.1 \%$ reduction in prevalence. This difference was statistically significant (McNemar Test $P<0.001$ ). Age group 9-10 years had the highest prevalence of infection 12 (2.9\%). Males recorded a higher prevalence of infection 21 (5.1\%) when compared to females 11 $(2.7 \%)(P>0.05)$. The mean urine egg count by sex at 6 months showed that males had a higher (1.99) mean urine egg count than females (1.97). The mean urine egg counts by sex at 12 months post treatment was 0.29 for males and 0.25 for females. There was $74.4 \%$ and $86.4 \%$ reduction in the mean egg count of the children at 6 and 12 months post treatment respectively while the mean urine egg count was reduced to 0.27 at 12 months from 1.98 at 6 months post treatment. At baseline, $4.4 \%$ of the participants had heavy egg infection (Fig. 2) which was reduced to $0.3 \%$ at 6 months post treat-

Table 1. Baseline prevalence of infection among the groups by sex and age

\begin{tabular}{|c|c|c|c|c|c|c|}
\hline \multirow{2}{*}{ Age groups (year) } & \multicolumn{2}{|c|}{ Male } & \multicolumn{2}{|c|}{ Female } & \multicolumn{2}{|c|}{ Both Sexes } \\
\hline & No. of examined & No. of Infected (\%) & No. of examined & No. of Infected (\%) & No. of examined & No. of Infected (\%) \\
\hline $5-6$ & 44 & 7 (15.9) & 31 & 4 (12.9) & 75 & $11(14.7)$ \\
\hline $7-8$ & 75 & $27(36.0)$ & 54 & $11(20.4)$ & 129 & $38(29.5)$ \\
\hline $9-10$ & 57 & 17 (29.8) & 48 & 15 (31.3) & 105 & $32(30.5)$ \\
\hline $11-12$ & 39 & $10(25.6)$ & 45 & $14(31.1)$ & 84 & 24 (28.6) \\
\hline $13-14$ & 17 & $1(5.9)$ & 12 & $0(0.0)$ & 29 & $1(3.4)$ \\
\hline$\geq 15$ & 12 & $2(16.7)$ & 0 & $0(0.0)$ & 12 & $2(16.7)$ \\
\hline Total & 244 & 64 (26.2) & 190 & 44 (23.2) & 434 & $108(24.9)$ \\
\hline
\end{tabular}




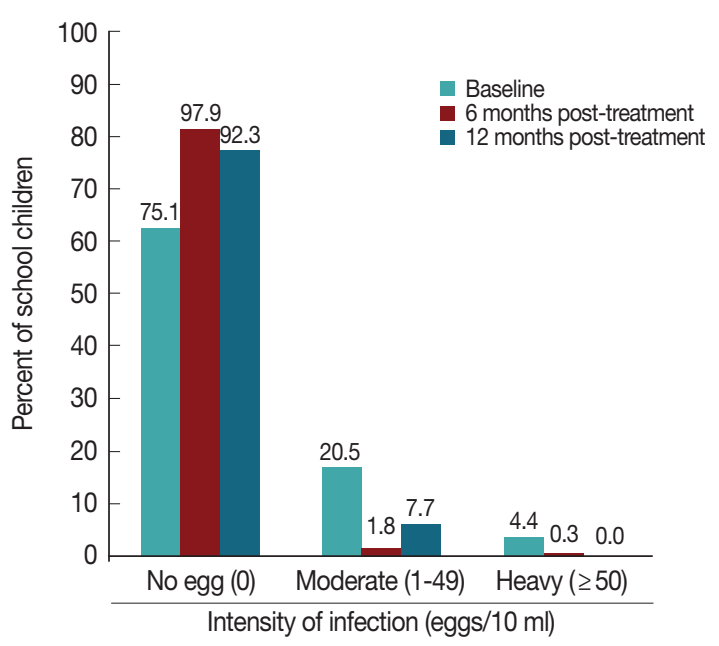

Fig. 2. Changes in Schistosoma haematobium egg intensity over a period of 12 months among pupils.

ment and eventually zero at 12 months post-treatment, though more participants (7.7\%) had moderate infection at 12 months than at 6 months post-treatment (1.8\%). The prevalence of visible haematuria was $1.8 \%$.

\section{DISCUSSION}

The infection of $S$. haematobium cuts across all age groups among the children and it could be noted that the older age groups such as age group 13-14 years had lower prevalence than most of the younger age groups. The baseline result showed that $S$. haematobium was endemic in the community though the influence of seasons could not be ascertained despite the fact that samples were collected at different times in 12 months though Tian-Bi et al. [21] identified infected snails exclusively during the dry season. The school with the lowest prevalence was the only private school in the community which had the children of the most enlightened people in the community, whereas the school with highest infection rate is a public school and probably the use of stream water for religious purpose could be the contributory factor to the high infection rate which showed the influence of practice on transmission. Single dose of praziquantel at $40 \mathrm{mg} / \mathrm{kg}$ body weight was able to significantly reduce the prevalence and intensity of infection uptill 6 months post-treatment. By the 12th month assessment of infection, there was an increased rate in the prevalence of infection among the children, some of whom were not found to be initially infected at baseline. This connotes new infection after treatment had started or some of the children harbor the juvenile stages of $S$. haematobium which according to Sabah et al. [22] are less susceptible to chemotherapy and could start oviposition by the 5th week of infection. Therefore, there is the need to support treatment activities with health education to avoid either re-infection or new infection of children [23]. Though, there was a significant increase in prevalence at 12th month post-treatment above the recorded 6th month prevalence, the intensity of infection was lower than the 6th month intensity. According to Toure et al. [16], the reduction in the intensity is particularly of importance as high intensity of $S$. haematobium infection has been shown to contribute to morbidity seen in schistosomiasis infection which includes anaemia in children [24]. Contrary to findings of Nsowah-Nuamah et al. [25] in a study in Ghana where the prevalence of infection was reduced by $80-90 \%$ at 12 months after treatment, the prevalence in the current study was reduced by $91.6 \%$ only for 6 months which was reduced to $69.1 \%$ by the 12 th month due to maturation of juvenile parasites that were not susceptible to the praziquantel [22] which have started oviposition and new infections. Therefore, our findings necessitate re-treatment with praziquantel after 12 months when prevalence increased contrary to the studies in Ghana [25] and Burkina Faso [16]. Although this is contrary to WHO [26] recommendation that communities with moderate risk ( $\geq 10 \%$ but $<50 \%$ prevalence) should treat once every 2 years. The uptrend of infection at the 12th month signified the need to constantly treat every year for achievement of elimination by year 2020. This will prevent a resurgence of infection to the pre-treatment level should there be an interruption in the drug distribution. Our present finding reveals the need for continuous efforts at monitoring the disease transmission and treatment coupled with other control measures such as snail control and health education. The current findings showed that WHO strategy of treatment once every 2 years could be adopted in some settings while there is need for some degree of flexibility in accordance to the epidemiological findings of other geographical areas.

\section{ACKNOWLEDGMENTS}

The project was funded by the Nigerian Institute of Medical Research. The authors appreciate the assistance of all the field assistants as well as the local guide. The cooperation and support of all the teachers and Head teachers of the schools and members of the community is hereby acknowledged. 


\section{CONFLICT OF INTEREST}

The authors have no conflict of interest to report.

\section{REFERENCES}

1. Cowper SG. Schistosomiasis in Nigeria. Ann Trop Med Parasitol 1963; 57: 307-322.

2. Colley DG, Bustinduy AL, Secor E, King CH. Human schistosomiasis Lancet 2014; 383: 2253-2264.

3. Steinmann P, Keiser J, Bos R, Tanner M, Utzinger J. Schistosomiasis and water resources development: systematic review, metaanalysis, and estimates of people at risk. Lancet Infect Dis 2006; 6: 411-425.

4. TchuemTchuenté L, Rollinson D, Stothard RJ, Molyneux D. Moving from control to elimination of schistosomiasis in subSaharan Africa: time to change and adapt strategies. Infect Dis Poverty 2017; 6: 42.

5. Molyneux DH, Savioli L, Engels D. Neglected tropical diseases: progress towards addressing the chronic pandemic. Lancet 2017; 389: 312-325

6. King $\mathrm{CH}$, Dangerfield-Cha $\mathrm{M}$. The unacknowledged impact of chronic schistosomiasis. Chronic Illn 2008; 4: 65-79.

7. Kjetland EF, Ndhlovu PD, Gomo E, Mduluza T, Midzi N, Gwanzura L, Mason PR, Sandvik L, Friis H, Gundersen SG. Association between genital schistosomiasis and HIV in rural Zimbabwean women. AIDS 2006; 20: 593-600.

8. Bustinduy A, King C, Scott J, Appleton S, Sousa-Figueiredo JC, Betson M, Stothard JR. HIV and schistosomiasis co-infection in African children. Lancet Infect Dis 2014; 14: 640-649.

9. Odaibo AB, Adewunmi CO, Olorunmola FO, Adewoyin FB, Olofintoye LK, Adewunmi TA, Adetula MO, Awe CO, Akinyemi F. Preliminary studies on the prevalence and distribution of urinary schistosomiasis in Ondo State, Nigeria. Afr J Med Med Sci 2004; 33: 219-224.

10. Hotez PJ, Asojo OA, Adesina AM. Nigeria: "Ground Zero" for the high prevalence neglected tropical diseases. PLoS Negl Trop Dis 2012; 6: e1600.

11. van der Werf MJ, Bosompem KM, de Vlas SJ. Schistosomiasis control in Ghana: case management and means for diagnosis and treatment within the health system. Trans R Soc Trop Med Hyg 2003; 97: 146-152.

12. Savioli L, Stansfield S, Bundy DA, Mitchell A, Bhatia R, Engels D, Montresor A, Neira M, Shein AM. Schistosomiasis and soiltransmitted helminth infections: forging control efforts. Trans R Soc Trop Med Hyg 2002; 96: 577-579.

13. Unicef. Water and sanitation monitoring platform Nigeria. Water and sanitation summary sheet. [Internet]; 2008. Available from: https://www.unicef.org/nigeria/NG_resources_washsummary.pdf.
14. Hotez PJ, Molyneux DH, Fenwick A, Kumaresan J, Sachs SE, Sachs JD, Savioli L. Control of neglected tropical diseases. N Engl J Med 2007; 357: 1018-1027.

15. Hotez PJ, Fenwick A, Savioli L, Molyneux DH. Rescuing the bottom billion through control of neglected tropical diseases. Lancet 2009; 373: 1570-1575.

16. Touré S, Zhang Y, Bosqué-Oliva E, Ky C, Ouedraogo A, Koukounari A, Gabrielli AF, Bertrand S, Webster JP, Fenwick A. Two-year impact of Single praziquantel treatment on infection in the National control programme on schistosomiasis in Burkina Faso. Bull World Health Organ 2008; 86: 780-787.

17. Hotez PJ. Mass drug administration and integrated control for the world's high prevalence neglected tropical diseases. Clin Pharmacol Ther 2009; 85: 659-664.

18. Rollinson D, Knopp S, Levitz S, Stothard JR, Tchuem Tchuenté LA, Garba A, Mohammed KA, Schur N, Person B, Colley DG, Utzinger J. Time to set the agenda for schistosomiasis elimination. Acta Trop 2013; 128: 423-440.

19. Hotez PJ, Fenwick A. Schistosomiasis in Africa: An emerging tragedy in our new global health decade. PLoS Negl Trop Dis 2009; 3: e485.

20. Mafe MA, von Stamm T, Utzinger J, N'Goran EK. Control of urinary schistosomiasis: an investigation into the effective use of questionnaires to identify high-risk communities and individuals in Niger State, Nigeria. Trop Med Int Health 2000; 5: 53-63.

21. Tian-Bi YT, Ouattara M, Knopp S, Coulibaly JT, Hürlimann E, Webster B, Allan F, Rollinson D, Meïté A, Diakité NR, Konan CK, N'Goran EK, Utzinger J. Interrupting seasonal transmission of Schistosoma haematobium and control of soil-transmitted helminthiasis in northern and central Côte d'Ivoire: a SCORE study protocol. BMC Public Health 2018; 18: 186.

22. Sabah AA, Fletcher C, Webbe G, Doenhoff MJ. Schistosoma mansoni: chemotherapy of infections of different ages. Exp Parasitol 1986; 61: 294-303.

23. World Health Organization. World Health Assembly Resolution (WHA) 66.12 Neglected tropical diseases. Geneva, Switzerland. World Health Organization. 2013.

24. World Health Organization. Prevention and Control of Schistosomiasis and Soil-Transmitted Helminthiasis. Report of a WHO Expert Committee. Geneva, Switzerland. World Health Organization. pp 1-57.

25. Nsowah-Nuamah NN, Aryeetey ME, Jolayemi ET, Wagatsuma $Y$, Mensah G, Dontwi IK, Nkrumah FK, Kojima S. Predicting the timing of second praziquantel treatment and its effect on reduction of egg counts in southern Ghana. Acta Trop 2004; 90: 263270.

26. World Health Organization. Preventive Chemotherapy in Human Helminthiasis: Coordinated Use of Anthelminthic Drugs in Control Interventions: A Manual for Health Professionals and Programme Managers. Geneva, Switzerland. World Health Organization. 2006. 
